\title{
Inversion Domain Boundaries in Wurtzite GaN
}

\author{
Joysurya Basu, R. Divakar, Sandeep Kumar, C. Barry Carter \\ Department of Chemical Engineering and Materials Science, 421 Washington Ave. SE, \\ University of Minnesota, Minneapolis, MN 55455, USA
}

Group-III nitrides are gaining increasing importance as optoelectronic materials with potential application in blue-UV light emitting diodes, lasers etc. In the family of groupIII nitrides $\mathrm{GaN}$ is particularly important. Because of the unavailability of a suitable substrate, a high defect density is always associated with GaN films grown on any substrate $[1,2]$, which degrades device performance and lifetime. GaN has a wurtzite structure with a polar $c$-axis. This structure is responsible for properties such as spontaneous polarization and Hall mobility used in devices. Of the many possible defects, inversion domain boundaries (IDB), across which cation and anion positions are interchanged, can be used to manipulate device performance. The structural origin of inversion domains in wurtzite $\mathrm{GaN}$ has always been explained with the $\{1010\}$ plane as the boundary and the atomic structure of any interface other than $\{10 \overline{1} 0\}$ is totally unknown. The motivation of this work is to characterize the structure and morphology of different IDBs in wurtzite GaN crystals.

Wurtzite GaN was grown by hydride vapor phase epitaxy on sapphire. The IDBs have been characterized using an FEI Tecnai T12 TEM operated at $120 \mathrm{kV}$. Diffraction contrast imaging and convergent beam electron diffraction have been used to understand the structure of the inversion domains.

In several $\mathrm{GaN}$ samples inversion domains in the form of pipes can be observed. The width of these domains is $\sim 50-100 \mathrm{~nm}$ and they are almost $1 \mu \mathrm{m}$ long. These domains show complementary contrast in the BF and DF image formed with the $\{10 \overline{1} 1\}$ reflections and they are invisible when imaged with the (1010) reflection. In another type of inversion domain, distinct boundaries can be observed. The domain shape is irregular and the boundaries facet along specific planes. The domain is invisible when imaged with $\{10 \overline{1} 0\}$ reflections indicating a possible translation vector along the [0001] axis. Further, the bounding planes in this domain are not edge on when the domain is oriented along the [0001] axis indicating that these boundaries are not of the $\{1010\}$ type. In convergent beam electron diffraction complementary contrast can be observed in the $\{1011\}$ disk and the thickness fringes change contrast across the boundary.

Three atomic models are available on the structure of inversion domain boundaries $[3,4]$. These are based on the interchange of cation and anion sub-lattices, filling up of a different set of tetrahedral voids across the boundary and a translation along the $c$-axis in order to avoid the $\mathrm{Ga}-\mathrm{Ga}$ and $\mathrm{N}-\mathrm{N}$ bonding, respectively. Notably, in all cases the boundary planes described are of the $\{10 \overline{1} 0\}$ type [5]. The results presented here show that these are inadequate to give a complete three-dimensional picture of IDBs, especially where the boundary facets on to a plane that is not of the $\{1010\}$ type. The combined 
results of diffraction contrast imaging and convergent beam electron diffraction will be interpreted to understand the structure of IDBs.

\section{References}

[1] R. F Davis et al. Acta mater., 51 (2003) 5961.

[2] J. K. Farrer et al. J. Mater. Sci. 41 (2006)

[3] S. B. Austerman and W. G. Gehman, J. Mater. Sci., 1 (1966) 249.

[4] D. B. Holt, J. Phys. Chem. Solids, 30 (1969) 1297.

[5] V. Potin et al., Phil. Mag. A, 79 (1999) 2899.

[6] This research is funded by the NSF through grant number NSF-DMR-0102327.
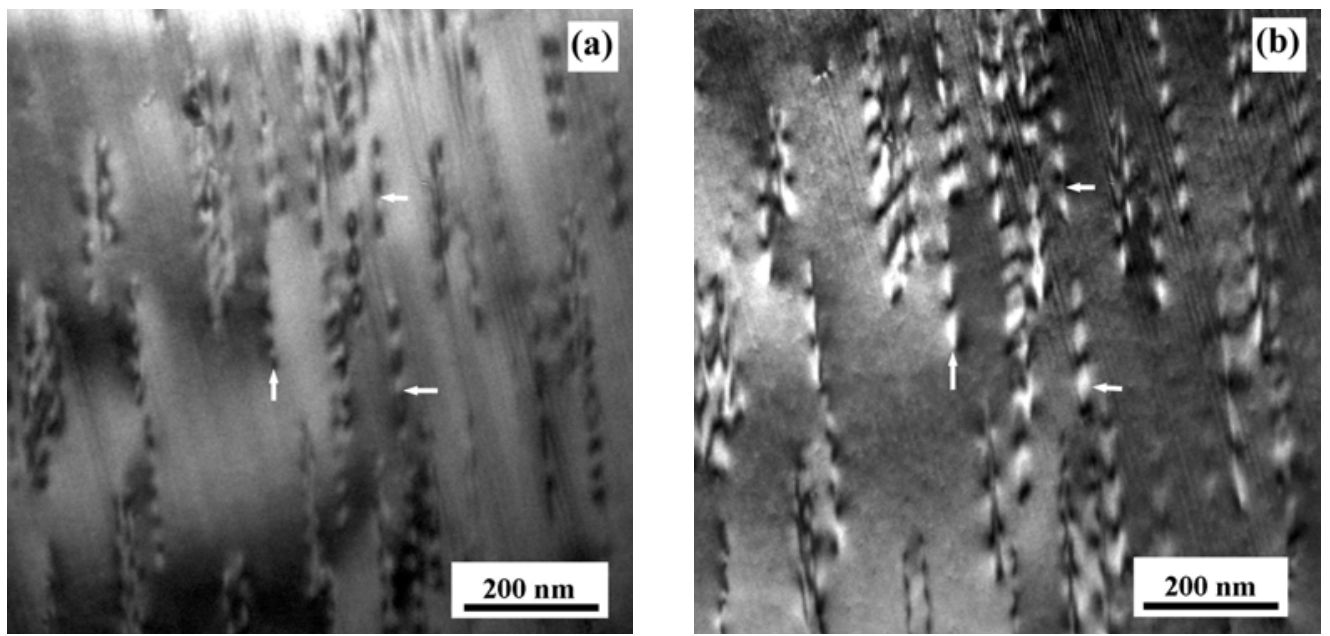

FIG. 1. Inversion domain pipes in GaN showing the complementary contrast in (a) bright field and (b) dark field.
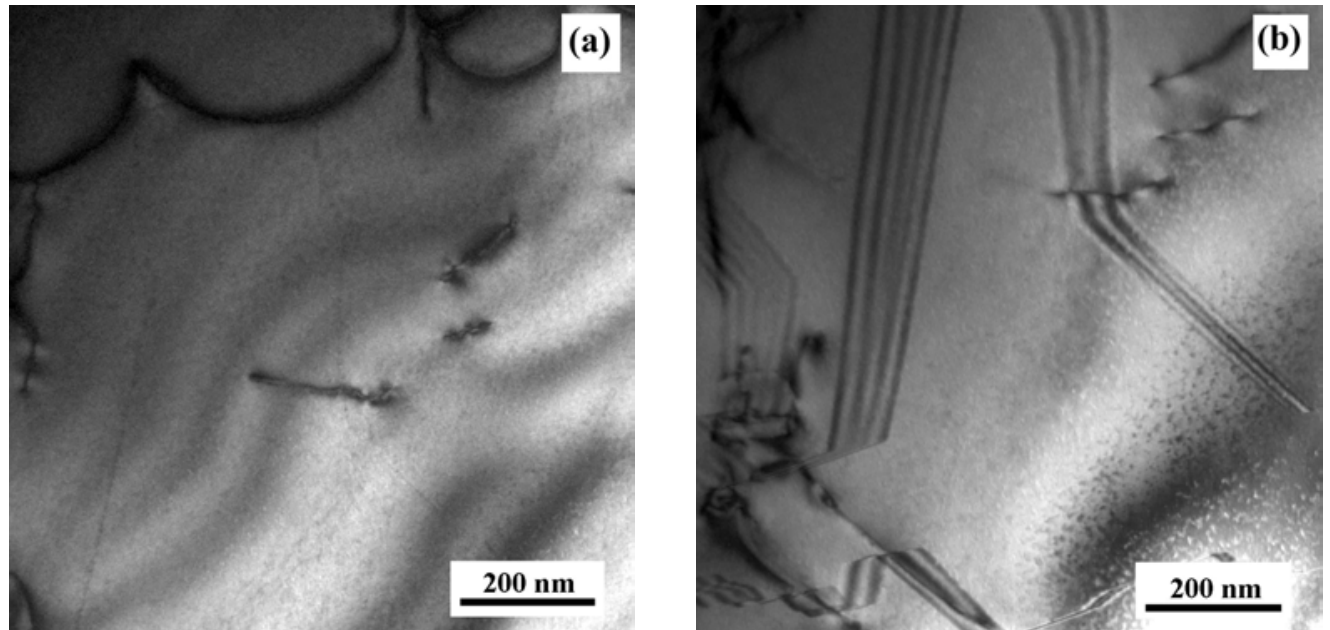

FIG. 2. Inversion domain boundary in GaN (a) the boundary is completely invisible with (1010), (b) the boundary is plane seen to facet on multiple planes including $\{10 \overline{1} 0\}$. 